\title{
Is there a common theme behind the correlated-electron superconductivity in organic charge-transfer solids, cobaltates, spinels and fullerides?
}

\author{
Sumit Mazumdar ${ }^{*}, 1$, R. Torsten Clay $^{2}$ \\ ${ }^{1}$ Department of Physics, University of Arizona, Tucson, AZ 85721, USA \\ ${ }^{2}$ Department of Physics and Astronomy and $\mathrm{HPC}^{2}$ Center for Computational Sciences, Mississippi State University, Mississippi State, \\ MS 39762, USA
}

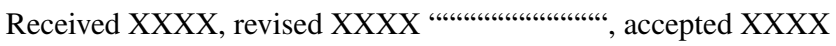

Published online XXXX

Key words: Strong correlations, Exotic superconductors

* Corresponding author: e-mail sumit@physics.arizona.edu, Phone: +001-520-621-6803, Fax: +001-520-621-4721

We posit that there exist deep and fundamental relationships between the above seemingly very different materials. The carrier concentration-dependences of the electronic behavior in the conducting organic charge-transfer solids and layered cobaltates are very similar. These dependences can be explained within a single theoretical model, - the extended Hubbard Hamiltonian with significant nearest neighbor Coulomb repulsion. Interestingly, superconductivity in the cobaltates seems to be restricted to bandfilling exactly or close to one-quarter, as in the organics. We show that dynamic Jahn-Teller effects and the resultant orbital ordering can lead to $\frac{1}{4}$-filled band descriptions for both superconducting spinels and fullerides, which show evidence for both strong electron-electron and electron-phonon interactions. The orbital orderings in antiferromagnetic lattice-expanded bcc $\mathrm{M}_{3} \mathrm{C}_{60}$ and the superconductor are different in our model. Strong correlations, quarter-filled band and lattice frustration are the common characteristics shared by these unusual superconductors.

Copyright line will be provided by the publisher

1 Introduction. The field of superconductivity (SC) is facing a crisis: 25 years after the discovery of high $\mathrm{T}_{c} \mathrm{SC}$, none of the proposed scenarios has led to a mechanism that scientists agree on. Although there is now general agreement that there exist many correlatedelectron superconductors, there is no understanding how any one scenario, proposed for one class of materials, could be extended to others. In the context of organic charge-transfer solids (CTS) alone, the natures of the insulating states proximate to SC can be quite different, including spin-density wave, antiferromagnetic, chargeordered or even a so-called valence bond solid. It is unlikely that the BCS theory, designed to explain the metal-to-superconductor transition, applies to any of these insulator-superconducting transitions. It is equally unlikely, however, that the mechanism of SC in structurally similar CTS, with closely related molecular components, are different for different initial insulating states, as has sometimes been proposed. We believe that determination of the characteristics shared by many seemingly different classes of superconducting materials will give the framework within which the theory of exotic SC should be constructed. Our goal here is to demonstrate that such a common framework might indeed exist for the CTS, inorganic layered cobaltates and spinels, and fullerides.

In the next section we examine the counterintuitive carrier density-dependent electronic behavior in the layered cobaltates. We point out that this behavior is very similar to that previously noted in the CTS, and show that both cobaltates and CTS can be understood within the same theoretical model, within which the bandfilling is a very important implicit parameter. It is then noteworthy that in both fam- 


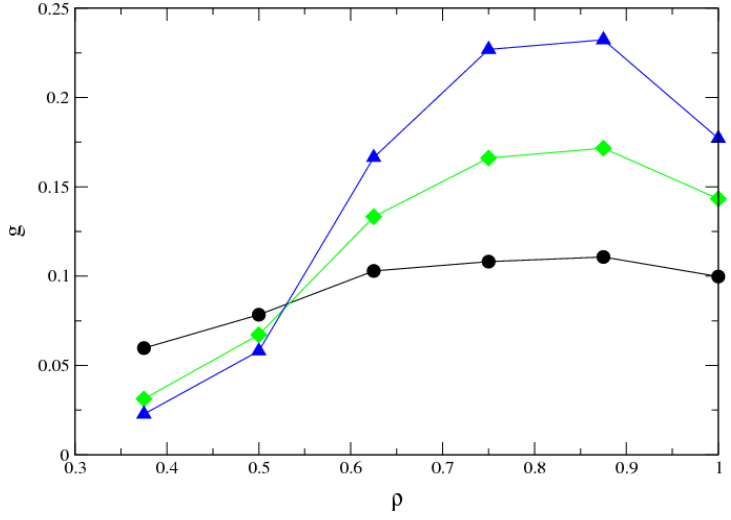

Figure 1 (Color online) Exact $g(\rho)$ versus $\rho$ for $U=10$, and $V=0$ (circles), 2 (diamonds) and 3 (triangles), for periodic ring of 16 sites. Lines are guides to the eye.

ilies SC appears to be limited to the $\frac{1}{4}$-filled band. Other features shared by the superconducting cobaltates and organics are strong Coulomb correlations and lattice frustration. We show that spinels and fullerides share the features common to the CTS and cobaltates. We suggest that for the strongly correlated frustrated $\frac{1}{4}$-filled band the Schafroth mechanism of SC [1] becomes operative.

2 Layered cobaltates and CTS - carrier density dependence. We are interested in $\mathrm{M}_{x} \mathrm{CoO}_{2}(\mathrm{M}=\mathrm{Li}, \mathrm{Na}$, $\mathrm{K})[2]$, the incommensurate "misfits" $\left[\mathrm{Bi}_{2} \mathrm{~A}_{2} \mathrm{O}_{4}\right]\left[\mathrm{CoO}_{2}\right]_{m}$ $(\mathrm{A}=\mathrm{Ca}, \mathrm{Sr}, \mathrm{Ba})[3]$ as well as the hydrated superconductor $\mathrm{Na}_{x} \mathrm{CoO}_{2}, y \mathrm{H}_{2} \mathrm{O}$ [4]. These materials consist of $\mathrm{CoO}_{2}$ layers, with the $\mathrm{Co}$-ions forming a triangular lattice, separated by layers of $\mathrm{M}^{+}$ions. The $\mathrm{Co}^{3+}$ and $\mathrm{Co}^{4+}$ ions are in their low-spin states because of large crystal field splitting [5]. Trigonal distortion splits the $t_{2 g}$ orbitals into low lying fully occupied doubly degenerate $e_{g}^{\prime}$ orbitals and higher energy $a_{1 g}$ orbitals that are occupied by one (in $\mathrm{Co}^{4+}$ ) or two (in $\mathrm{Co}^{3+}$ ) electrons [6]. Only the $a_{1 g}$ orbitals are relevant for transport and thermodynamics. Charge carriers are $S=\frac{1}{2}$ holes on the $\mathrm{Co}^{4+}$ [5], and hence the carrier density $\rho=1-x$ in $\mathrm{M}_{x} \mathrm{CoO}_{2}$.

The onsite repulsion Hubbard $U$ is large in cobaltates [2]. Simplistically, large $\rho<1$ is close to the $\rho=1$ MottHubbard limit and should behave as strongly correlated; small $\rho$ should behave as weakly correlated as it is close to the $\rho=0$ band semiconductor limit (all Co-ions trivalent.) Experiments probing magnetic susceptibility and thermoelectric power have, however, demonstrated that the actual $\rho$-dependence is exactly the opposite; in the experimentally accessible range $\rho=0.2-0.8$, small (large) $\rho$ is strongly (weakly) correlated [2,3]. The basic observation is the same for all $\mathrm{M}_{x} \mathrm{CoO}_{2}$ and the misfits, indicating that $\rho$-dependence is intrinsic to the $\mathrm{CoO}_{2}$ layer.

A hint to the understanding of cobaltates comes from prior observation on quasi-one-dimensional (quasi-1D) conducting CTS [7], in which $\rho$ ranges from 0.5 to 1 . CTS (a)
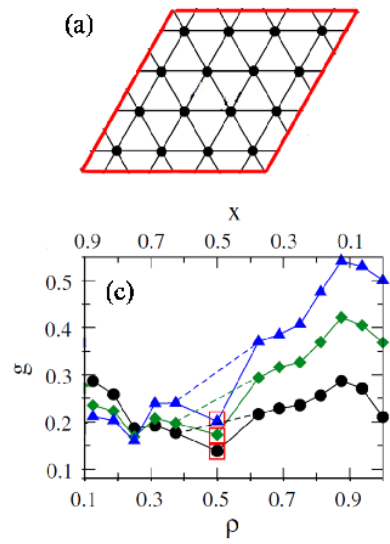

(b)

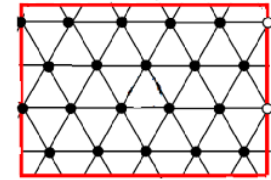

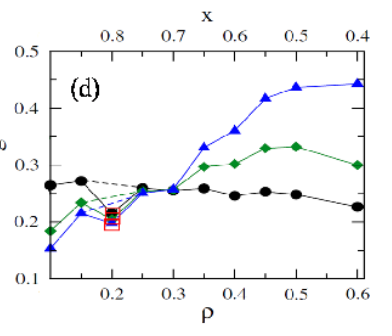

Figure 2 (Color online) (a) and (b) $N_{s}=16$ and 20-site clusters investigated numerically. (c) and (d) Exact $g(\rho)$ versus $\rho$. The circles, diamonds and triangles correspond $V=0,2$ and 3, respectively. The boxes correspond to data points with total spin $S>S_{\min }=0\left(\frac{1}{2}\right)$ for even (odd) numbers of particles.

with $\rho$ equal to or close to 0.5 exhibit magnetic susceptibility enhanced relative to the Pauli susceptibility, and lattice instability with periodicity $4 \mathrm{k}_{F}$ (where $\mathrm{k}_{F}$ is the Fermi wavevector within one-electron theory), both accepted as signatures of strong correlations. In contrast, $\rho$ between 0.66 and 0.8 show weakly correlated behavior, viz., unenhanced susceptibility and the usual $2 \mathrm{k}_{F}$ Peierls instability (see Tables in reference [7]). The behavior in the CTS and the cobaltates are then very similar.

3 Theory of carrier density-dependent electronic behavior. We present here a theory of the $\rho$-dependent electronic behavior, in 1D [7] and the 2D triangular lattice [8]. Consider the extended Hubbard Hamiltonian,

$$
H=-\sum_{\langle i j\rangle \sigma} t_{i j} c_{i \sigma}^{\dagger} c_{j \sigma}+U \sum_{i} n_{i, \uparrow} n_{i, \downarrow}+V \sum_{\langle i j\rangle} n_{i} n_{j}
$$

where $c_{i \sigma}^{\dagger}$ creates an electron or a hole with spin $\sigma$ ( $\uparrow$ or $\downarrow$ ) on site $i,\langle\ldots\rangle$ implies nearest neighbors (NN), and all other terms have their usual meanings. Individual molecules (Co-ions) are the sites in the CTS (cobaltates).

Strongly correlated behavior, at any $\rho$, requires that the ground state wavefunction has relatively small contribution from configurations with double occupancies. We therefore calculate numerically the normalized probability of double occupancy in the ground state

$$
g(\rho)=\frac{\left\langle n_{i, \uparrow} n_{i, \downarrow}\right\rangle}{\left\langle n_{i, \uparrow}\right\rangle\left\langle n_{i, \downarrow}\right\rangle}
$$

$g(\rho)$ is clearly weakly $\rho$-dependent for $V=0$. For $V>0$ and $\rho=0.5$, electrons are prevented from encountering one another by $V$, thus reducing $g(\rho)$ more than $U$ alone. 

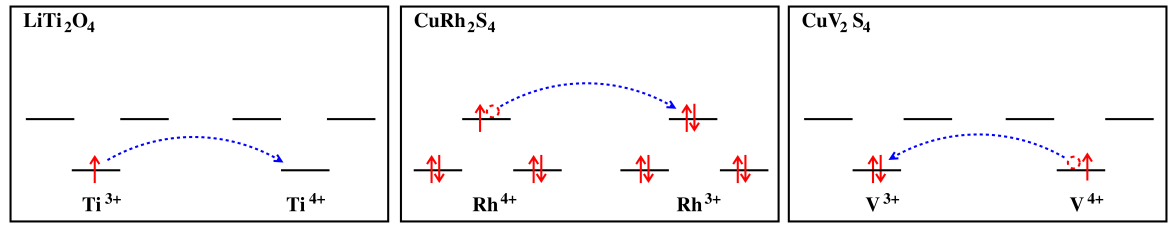

Figure 3 (Color online) The effective $\frac{1}{4}$-filled band natures of the superconducting spinels, following the splitting of the $t_{2 g}$ orbitals (see text). The blue dotted arrows denote electron or hole motion.

As $\rho$ is increased from 0.5 , more and more intersite repulsions are generated with additions of electrons, and configurations with a few double occupancies begin to compete with those with only singly occupied sites. Thus $g(\rho)$ increases steeply with increase in $\rho$ for significant $V / U$. As $\rho$ increases beyond an intermediate range, the Mott-Hubbard limit is approached and $g(\rho)$ should decrease again.

Exact numerical calculations of $g(\rho)$ verify this conjecture. Our earlier 1D calculations were for relatively small numbers of electrons $N_{e}$ with varying number of sites $N_{s}$ [7]. Significantly larger computer capabilities now allow calculations for different $\rho$ with fixed $N_{s}=16$. In Fig. 1 we have shown the results of our calculations for $U /|t|=$ 10 and $V /|t|=0,2$ and 3 . This value of $U$ is considered realistic for CTS, and there is evidence for $V /|t|$ as large as 3. The plots are similar for $U /|t|=6-12$ and $V /|t|=1-3$. While CTS with $\rho<0.5$ do not exist, we have included this smaller $\rho$ for comparison to 2D. We conclude from Fig. 1 that for realistic $V / U$, strong $\rho$-dependent electronic behavior is warranted. Experimentally [7], all $\rho=0.5$ CTS (e.g., MEM(TCNQ) $)_{2}$, Qn(TCNQ $)_{2}$, (TMTTF $)_{2}$ X, etc.) exhibit strongly correlated behavior, while CTS with $\rho=$ $0.66-0.8$ (TTFBr $_{0.74}$, HMTTF- and HMTSF-TCNQ with $\rho=0.75$, etc.) uniformly exhibit weakly correlated behavior. Subsequent demonstrations of strongly correlated behavior in 2:1 BEDT-TTF [9] or 1:2 $\mathrm{Pd}(\mathrm{dmit})_{2}$ systems [10] further support the theory.

In Fig. 2 we show our results for the finite periodic 2D triangular lattices with $N_{s}=16$ (Fig. 2(a)) and 20 (Fig. 2(b).) We have taken $t>0$. Elsewhere we have argued that the $U /|t|$ and $V /|t|$ in the cobaltates are similar to that in the CTS [8]. With the exception of a few $N_{e}$, the ground state is always in the lowest spin state $\left(0\left(\frac{1}{2}\right)\right.$ for $N_{e}=$ even(odd)) for $t>0$. As seen in Figs.2(c) and (d) the behavior of $g(\rho)$ in 2D is similar to that in 1D, and explains the much-discussed lack of symmetry about $\rho=0.5$ in the cobaltates - strongly correlated behavior near $\rho=\frac{1}{3}$ and weakly correlated behavior near $\rho=\frac{2}{3}$ [2].

4 Superconductivity in the CTS and layered cobaltates. We point out that SC is limited to the same narrow range of carrier concentration in both families. Given the strong role of $\rho$, this cannot but be significant. CTS superconductors are 2:1 cationic or 1:2 anionic materials [11], with $100 \%$ charge-transfer, and consequently $\rho=0.5$. The lattice structures of the superconducting CTS are not quasi-1D but anisotropic triangular, giving both frustration and larger bandwidth.
In the hydrated superconducting Na-cobaltate, $x \simeq$ 0.35 , Careful experiments have revealed however that $\rho$ is considerably smaller than 0.65 , as a portion of the water enters as $\mathrm{H}_{3} \mathrm{O}^{+}$. A significant number of investigators have determined that $\rho$ in the superconductor is exactly or close to 0.5 [12]. It is also noteworthy that $x=0.5$ is unique, Hall and Seebeck coefficients change signs only at this $\rho$ at low temperature in both $\mathrm{Na}_{x} \mathrm{CoO}_{2}$ and $\mathrm{Li}_{x} \mathrm{CoO}_{2}$.

The limitation of SC to $\rho=0.5$ acquires additional significance when taken together with: (a) absence of SC in the $\rho=1$ triangular lattice Hubbard Hamiltonian [13], (b) the propensity at $\rho=0.5$ to form the paired-electron crystal (PEC), in which spin-singlet pairs are separated by pairs of vacant sites, in both $1 \mathrm{D}$ and the moderately frustrated anisotropic triangular lattice [14]. We have proposed that with further increase in frustration the PEC gives way to a superconducting paired-electron liquid [14]. The proposed theory of SC is essentially the same as that of Schafroth, within which electron-pairs form mobile molecules (charged bosons) [1]. It is, however, different from the mean-field theory of charge-fluctuation mediated SC [15]. In the rest of this paper we show that there exist other systems which likely can be understood within the same scenario.

5 Superconducting spinels. Spinels are inorganic ternary compounds $\mathrm{AB}_{2} \mathrm{X}_{4}$, where the $\mathrm{X}^{2-}$ form a closepacked structure with the $A(B)$ sites occupying tetrahedral (octahedral) interstices. The B-cations are the active sites, with small X-mediated B-B electron hoppings. The B sublattice is tetrahedral, and hence frustrated.

$\mathrm{LiTi}_{2} \mathrm{O}_{4}, \mathrm{CuRh}_{2} \mathrm{~S}_{4}$ and $\mathrm{CuRh}_{2} \mathrm{Se}_{4}$ are the only three spinels that have been confirmed to be superconductors; reports of $\mathrm{SC}$ in $\mathrm{CuV}_{2} \mathrm{~S}_{4}$ and $\mathrm{CoCo}_{2} \mathrm{~S}_{4}$ exist. $\mathrm{SC}$ in $\mathrm{LiTi}_{2} \mathrm{O}_{4}$ was discovered many years before the cuprates [16], and has been of interest ever since because of its high $\mathrm{T}_{c} / \mathrm{T}_{F}$ ( $T_{F}$ is Fermi temperature), which is closer to that in the cuprates than conventional superconductors [17]. The mechanism of SC remains controversial: strong coupling BCS theory, the RVB approach [18] and the bipolaron theory [19] of SC have all been proposed.

Examination of the common valence of the B-cations in the superconducting spinels, 3.5+, reveals remarkable similarities between them and the CTS and cobaltates. The $\mathrm{Ti}^{3.5+}$ ions in $\mathrm{LiTi}_{2} \mathrm{O}_{4}$ have one $d$-electron per two Ti. $\mathrm{Rh}^{3.5+}$ and $\mathrm{Co}^{3.5+}$ in their low-spin states possess one $d$-hole per two metal ions. $\mathrm{V}^{3+}$ and $\mathrm{V}^{4+}$ have electron configurations $3 \mathrm{~d}^{1}$ and $3 \mathrm{~d}^{2}$, respectively. In all cases static band Jahn-Teller lattice distortions will give $\frac{1}{4}$-filled elec- 
tron (in $\mathrm{LiTi}_{2} \mathrm{O}_{4}$ ) and hole (in $\mathrm{CuRh}_{2} \mathrm{~S}_{4}, \mathrm{CoCo}_{2} \mathrm{~S}_{4}$ and $\mathrm{CuV}_{2} \mathrm{~S}_{4}$ ) bands as indicated in Fig. 3. Static lattice distortions should give a 3D PEC, as indeed observed in $\mathrm{CuIr}_{2} \mathrm{~S}_{4}$ [20] and $\mathrm{LiRh}_{2} \mathrm{O}_{4}$ [21]. We propose that the superconducting state is a 3D paired-electron liquid, with dynamical (as opposed to static) orbital ordering.

6 Superconducting fullerides. The $\mathrm{C}_{60}^{3-}$ ions in superconducting $\mathrm{M}_{3} \mathrm{C}_{60}$ form fcc lattices. Theories of fullerides treat each $\mathrm{C}_{60}$ unit as a site, with electron hoppings between the triply degenerate $t_{1 u}$ antibonding molecular orbitals. Although many experiments suggest onsite pairing mediated by $H_{g}$ Jahn-Teller phonons [22], the large $\mathrm{T}_{C} / \mathrm{T}_{F}$ suggests a non-BCS mechanism [17]. Antiferromagnetism in bcc $\mathrm{Cs}_{3} \mathrm{C}_{60}$ also indicates strong Coulomb repulsion [23,24]. Nonzero spin gap to the lowest energy high-spin state in the antiferromagnet has indicated that it is the effective $\frac{1}{2}$-filled band Mott-Hubbard insulator of Fig. 4(a), reached after Jahn-Teller distortion [23,24].

Within a dynamic mean field theory (DMFT) of the pressure-induced antiferromagnetism-to-SC in $\mathrm{Cs}_{3} \mathrm{C}_{60}$ [24], pairing arises from the combined effects of Hubbard $U$ and Jahn-Teller interaction within the effective $\frac{1}{2}$-filled band of Fig. 4(a), at the interface of an insulating and a metallic phase. We recall that the DMFT approach to $\mathrm{SC}$ in the CTS within the effective $\frac{1}{2}$-filled band Hubbard model was very similar. It is now known that SC in this latter case was an artifact of the mean-field approximation [13]. We believe that a similar criticism applies also to the existing theory of SC in the fullerides.

The difficulty in arriving at a theory of SC arises from the bias that the orbital occupancies are the same in the antiferromagnetic and superconducting phases. We suggest that pressure induces a new orbital ordering, with $\mathrm{C}_{60}^{3-}$ configurations as shown in Fig. 4(b), where two degenerate $\frac{1}{4}$-filled bands bands are obtained. This orbital "reordering" will be driven by the lower total energy of two $\frac{1}{4}$-filled bands compared to that of the single effective $\frac{1}{2}$-filled band, for larger bandwidth. Each of the $\frac{1}{4}$-filled bands can now form its own paired-electron liquid.

7 Conclusion. Our goal here was to show that the correlated $\frac{1}{4}$-filled band offers a common description of seemingly very different classes of materials as well as a plausible mechanism of SC. The key step is the realization that antiferromagnetism in the semiconducting state does not necessarily mean that the electronic structure of the superconductor is derived from the same configuration. Bandwidth-driven transition to a different state that is more appropriately described as $\frac{1}{4}$-filled can occur in the superconducting state [14]. This has been explicitly shown in the context of the CTS. Work is in progress to demonstrate the same within the models of Figs. 3 and 4. The proposed mechanism offers, (a) a way to arrive at a single unified theory of SC for the CTS, with different kinds of proximate insulating states, and (b) understanding of the strong role of electron-phonon interactions, and yet large $\mathrm{T}_{c} / \mathrm{T}_{F}$, in the spinels and the fullerides. In both spinels and fullerides, dynamic Jahn-Teller phonons will play a strong role in the correlated superconductor. However, this is not a signature of BCS pairing.

Acknowledgements This work was partially supported by DOE Grant No. DE-FG02-06ER46315.

\section{References}

[1] M. R. Schafroth, Phys. Rev. 100, 463 (1955).

[2] Y. Wang et al., Nature 423, 425 (2003). M. L. Foo et al., Phys. Rev. Lett. 92, 247001 (2004). T. Motohashi et al., ibid, 83, 195128 (2011).

[3] V. Brouet et al., Phys. Rev. B, 76, 100403(R) (2007).

[4] K. Takada et al., Nature (London) 422, 53 (2003).

[5] M. Z. Hasan et al., Phys. Rev. Lett. 92, 246402 (2004).

[6] T. Mizokawa, New J. Phys. 6, 169 (2004).

[7] S. Mazumdar and A. N. Bloch, Phys. Rev. Lett. 50, 207 (1983). S. Mazumdar and S. N. Dixit, Phys. Rev. B 34, 3683 (1986).

[8] H. Li, R. T. Clay and S. Mazumdar, Phys. Rev. Lett. 106, 216401 (2011).

[9] K. Kanoda, J. Phys. Soc. Jpn. 75, 051007 (2006).

[10] R. Kato, Chem. Rev. 104, 5319 (2004).

[11] G. Saito, K. Yamaji and T. Ishiguro, Organic Superconductors (Springer-Verlag, 1988).

[12] P. W. Barnes et al. Phys. Rev. B 72, 134515 (2005). H. Sakurai et al., ibid, 74, 092502 (2006). M. Bañobre-López et al., J. Am. Chem. Soc. 131, 9632 (2009). T. Shimojima et al., Phys. Rev. Lett. 97, 267003 (2006).

[13] R. T. Clay, H. Li and S. Mazumdar, Phys. Rev. Lett. 101, 166403 (2008).

[14] R. T. Clay et al., see present proceedings.

[15] J. Merino and R. H. McKenzie, Phys. Rev. Lett. 87, 237002 (2001).

[16] D. C. Johnston, J. Low Temp. Phys. 25, 145 (1976).

[17] W. D. Wu et al., Hyperfine Interactions, 86, 615 (1994); Y. J. Uemura, Physica C 185-189, 733 (1991).

[18] P. W. Anderson et al. Phys. Rev. Lett. 58, 2790 (1987).

[19] A. Alexandrov and J. Ranninger, Phys. Rev. B 24, 1164 (1981).

[20] P. G. Radaelli et al., Nature 416, 155 (2002).

[21] Y. Okamoto et al., Phys. Rev. Lett. 101, 086404 (2008).

[22] C. M. Varma, J. Zaanen and K. Raghavachari, Science 254. 989 (1991).

[23] Y Iwasa and T Takenobu, J. Phys. Cond. Matter, 15 R495 (2003).

[24] M. Capone et al., Rev. Mod. Phys. 81, 943 (2009). 
(a)

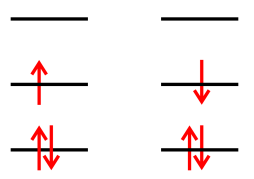

(b)

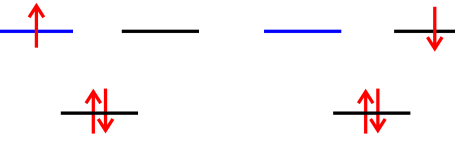

Figure 4 (Color online) (a) The effective $\frac{1}{2}$-filled $t_{1 u}$ band in antiferromagnetic $\mathrm{C}_{60}^{3-}$, following Jahn-Teller distortion. (b) The proposed doubly degenerate $\frac{1}{4}$-filled bands in the superconductor (see text). 